\title{
Focus on surface analysis
}

\author{
Michael Kopnarski
}

Published online: 18 March 2011

(C) Springer-Verlag 2011

Since the early 1970s, surface, interface and thin film analysis has evolved from surface science and has become a rapidly developing area in research and technology. This type of analysis, briefly referred to as surface analysis, provides insight into the chemical and structural properties of surfaces and thin films and clearly distinguishes the surface characteristics from those of the underlying bulk. The particular challenge of this kind of analysis arises from the fact that the chemical or structural analysis has to be accompanied by the precise determination of the related analysis volume. This, however, requires ultimate sensitivity, because at least in the case of high spatial resolution, the dimension of this volume is in the nanometre range.

Although surface analysis found its way into industrial practice long ago, the development of application-oriented surface analytical solutions also taking into account the corresponding economic conditions is still relevant today. However, it is frequently overlooked that surface analysis is not only used in other scientific or technological fields, but also represents in its own right an interdisciplinary and independent scientific field with a strategic cross-sectional function. Nanotechnology, which is inseparably connected to the discovery and development of scanning probe techniques such as scanning tunnelling microscopy and atomic force microscopy, is a good example for this assertion.

It was through the interplay between application, instrumental development and basic research that advances

Published in the special issue Applied Surface Analysis with Michael Kopnarski as Guest Editor.

\section{Kopnarski $(\bowtie)$}

IFOS $\mathrm{GmbH}$,

Trippstadter Straße 120,

67663 Kaiserslautern, Germany

e-mail: kopnarski@ifos.uni-kl.de became possible. The advent of delay line detectors facilitated the development of quantitative tomographic atom probes with spatial resolution down to the atomic scale and significantly improved the so-called parallel imaging in photoelectron spectroscopy as well. And vice versa, the demands of other areas of research, nanotechnology and life sciences for instance, initiated new efforts in surface analytics. In the field of secondary ion mass spectrometry, the introduction of high-performance time-offlight mass spectrometers and advanced ion sources $\left(\mathrm{SF}_{5}{ }^{+}\right.$, $\mathrm{Bi}_{n}{ }^{+}, \mathrm{C}_{60}{ }^{+}$, etc.) led to improvements in sensitivity and lateral resolution and has even allowed sputter depth profiling of organic molecules containing information exploitable for surface chemical identification and distribution analysis. Thus, this opens up entirely new potentialities in bioanalytical applications, for instance in the pharmaceutical area (drugs, proteins, etc.).

The special part of this issue is focused on surface and depth profile analysis and reflects the above-mentioned aspects very well. Surface analysis, to use the words of Wilhelm Ostwald, may be the kitchen maid or the lady of science; it is in the eye of the beholder.

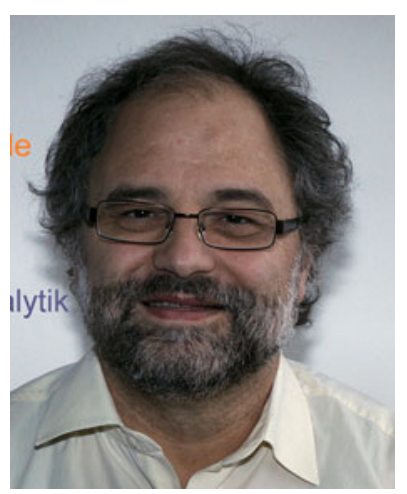

Michael Kopnarski

is currently a member of the Research Centre for Optics and Material Science OPTIMAS and the head of the Institute of Surface and Thin Film Analysis IFOS at the Technical University of Kaiserslautern. His main research interests are instrumental and applied surface and thin film analysis. 